\title{
THE INEQUALITIES OF MORSE WHEN THE MAXIMUM TYPE IS AT MOST THREE ${ }^{1}$
}

\author{
ROSS H. BARDELL
}

1. Introduction. The theory of critical points has been developed by $\operatorname{Morse}^{2}[1,2]$ by the use of combinatorial topology. In particular the theory developed by Morse is applicable to simple integral problems in the calculus of variations. The author, in his doctoral dissertation [3], studied the theory of types of extremals for a simple integral problem in the plane without the use of combinatorial topology. In the present paper a new and interesting property of extremal arcs joining two fixed points in the plane will be proved. This property makes possible a proof of the inequalities of Morse [3, p. 30] in the special case where the maximum type of each extremal arc is at most three. This is the first proof of the inequalities without the use of topology and without assuming the problem to be reversible [3, p. 25].

The hypotheses of this paper are those made in $\$ 1$ of the dissertation [3] referred to above and likewise the notation used here is that of the earlier paper.

2. Properties of the extremal arcs joining two fixed points of $R$. In the paper mentioned above [3, p. 17] it was proved that every point 2 of $R$, which is not on an envelope arc of the family of extremal arcs though the point 1 , is joined to 1 by $2 r+1$ ( $r$ a positive integer) extremal arcs of which $r$ are of odd type and $r+1$ are of even type, one of which at least is of type zero.

Consider now a point 2 of $R$ which is not on an envelope arc of the family of extremals through the point 1 . Let $E_{a_{0}}$ designate an extremal arc joining the point 2 to 1 and such that the arc 12 of $E_{a_{0}}$ is of type zero. It can be shown [3, p. 10] that the extremal arc $E_{a}$ for $a>a_{0}$ and near $a_{0}$ and also for $a<a_{0}+2 \pi$ and near $a_{0}+2 \pi$ has no intersections with the arc 12 of $E_{a_{0}}$. Then as $a$ increases from $a_{0}$ to $a_{0}+2 \pi$ all intersections of the extremal arc $E_{a}$ with the arc 12 of $E_{a_{0}}$ which move onto this arc must move off again. In fact it can easily be proved [3, p. 18] that an intersection 3 of $E_{a}$ with the arc 12 of $E_{a_{0}}$ which moves onto the arc 12 of $E_{a_{0}}$ for an extremal arc of odd (even) type must move off of the arc 12 of $E_{a_{0}}$ for an extremal arc of even

${ }^{1}$ Presented to the Society, April 8, 1938.

2 The numbers in brackets here and elsewhere refer to the bibliography at the end of this paper. 
(odd) type. While the preceding statement is true for all extremal arcs, the following much stronger property can be proved in the special case where the extremal arcs in question are of type zero or one.

LEMмA 2.1. Let 2 be any point of the region $R$ which is not on an envelope arc of the family of extremals through 1 , and let 3 designate a particular intersection of $E_{a}$ with the arc 12 of $E_{a_{0}}$ which is moving through 2 onto the arc 12 of $E_{a_{0}}$ as a increases through $a^{\prime}$ and off of the arc 12 of $E_{a_{0}}$ as a increases through $a^{\prime \prime}$, where $a^{\prime}<a^{\prime \prime}$. Then, if the arc 12 of $E_{a^{\prime}}$ is of type zero, the arc 12 of $E_{a^{\prime \prime}}$ is of type one. Moreover, if the arc 12 of $E_{a^{\prime \prime}}$ is of type zero, then the arc 12 of $E_{a^{\prime}}$ is of type one.

It will suffice to prove the first statement of the above lemma since the last statement is exactly equivalent to the first when the parameter is allowed to decrease from $a^{\prime \prime}$ to $a^{\prime}$. We will now proceed to prove the first statement.

Since, by hypotheses, the arc 12 of $E_{a^{\prime}}$ is of type zero, it follows that the arc 13 of $E_{a}$ for $a>a^{\prime}$ and near $a^{\prime}$ has no intersections with the arc 12 of $E_{a^{\prime}}$ [3, p. 10]. Moreover, as $a$ increases from $a^{\prime}$ to $a^{\prime \prime}$ the point 3 never passes through 2 , and hence no intersection of the arc 13 of $E_{a}$ can move onto the arc 12 of $E_{a^{\prime}}$. Therefore the arc 13 of $E_{a}$ for $a^{\prime}<a<a^{\prime \prime}$ has no intersections with the arc 12 of $E_{a^{\prime}}$ and hence the arc 12 of $E_{a^{\prime \prime}}$ has only the point 2 in common with the arc 12 of $E_{a^{\prime}}$.

Let the arc 12 of $E_{a^{\prime \prime}}$ be of type $k$. Then as a decreases from $a^{\prime \prime}$ and is near $a^{\prime \prime}$, the arc 13 of $E_{a}$ will have $k$ intersections with the arc 12 of $E_{a^{\prime \prime}}$. We wish to show that the number of intersections of the arc 13 of $E_{a}$ with the arc 12 of $E_{a^{\prime \prime}}$ is also equal to $k$ for $a \leqq a^{\prime}$ and near $a^{\prime}$. In that case the preceding paragraph shows that $k=1$ and hence the lemma is proved. To show this let 4 and 6 designate the intersections of $E_{a^{\prime}}$ and $E_{a^{\prime \prime}}$, respectively, with $E_{a_{0}}$ which are adjacent to and preceding 2 on $E_{a_{0}}$ and let 5 designate the intersection of $E_{a}$ with $E_{a_{0}}$ which is adjacent to and preceding 3 on $E_{a_{0}}$. Further let the intersection of $E_{a^{\prime \prime}}$ with $E_{a_{0}}$ which is adjacent to and preceding 6 on $E_{a_{0}}$ be designated by 7 . We have then the following two cases to consider.

CASE 1. Sin $\left(\theta_{0}-\theta\right)$ has opposite signs at the two points 4 and 2, where $\theta_{0}$ and $\theta$ are the inclinations of the tangents to $E_{a_{0}}$ and $E_{a}$, respectively, at their particular intersection in question. In this case the intersections 5 and 3 can move only on the interval 42 of $E_{a_{0}}$ for $a^{\prime}<a<a^{\prime \prime}$. This follows since, as was seen above, the arc 13 of $E_{a}$ has no intersections with the arc 12 of $E_{a^{\prime}}$, for $a^{\prime}<a<a^{\prime \prime}$. Therefore the intersection 6 must also be on the interval 42 of $E_{a_{0}}$ and by exactly 
the same reasoning it follows that the intersection 7 must be on the interval 14 of $E_{a_{0}}$. Hence as $a$ decreases from $a^{\prime \prime}$ to $a^{\prime}$ the intersection 3 , which must stay on the interval 42, may pass through 6 but not through 7. When it does pass through 6 an intersection of the arc 13 of $E_{a}$ with the arc 12 of $E_{a^{\prime \prime}}$ is lost, but is regained when 3 reverses its direction and moves again through 6 as it must since its final position is 2 . Therefore in this case the arc 13 of $E_{a}$ for $a \leqq a^{\prime}$ and near $a^{\prime}$ has $k$ intersections with the arc 12 of $E_{a^{\prime \prime}}$.

CASE 2. Sin $\left(\theta_{0}-\theta\right)$ has the same sign at the two intersections 4 and 2 . In this case the point 3 can move only on the interval 42 of $E_{a_{0}}$ and the point 5 can move only on the interval 14 of $E_{a_{0}}$ as $a$ decreases from $a^{\prime \prime}$ to $a^{\prime}$. This follows from the fact that, as above, the arc 13 of $E_{a}$ has no intersections with the arc 12 of $E_{a^{\prime}}$ for $a^{\prime}<a<a^{\prime \prime}$. Therefore 6 must remain on the interval 14 of $E_{a_{0}}$ as $a$ decreases from $a^{\prime \prime}$ to $a^{\prime}$ and hence 3 cannot pass through 6 since 3 must remain on the interval 42 of $E_{a_{0}}$. Hence in this case also the arc 13 of $E_{a}$ for $a^{\prime} \leqq a<a^{\prime \prime}$ always has $k$ intersections with the arc 12 of $E_{a^{\prime \prime}}$.

3. The inequalities. For every $a$ on the interval $a_{0}<a<a_{0}+2 \pi$ defining an extremal arc $E_{a}$ which joins the point 2 to 1 an intersection of $E_{a}$ with the arc 12 of $E_{a_{0}}$ either moves onto or off of this arc. Let the extremal arcs joining the points 1 and 2 be paired so that the arcs of each pair are those defined in the family of extremals through the point 1 by the parameter values for which a particular intersection 3 of $E_{a}$ with the arc 12 of $E_{a_{0}}$ moves onto and off of this latter arc.

LEMMA 3.1. Every extremal arc of the set of extremals joining the points 1 and 2, except $E_{a_{0}}$, belongs to one and only one of the pairs of extremal arcs defined above. Moreover, if the maximum type of each extremal arc joining the points 1 and 2 is assumed to be not greater than 3, then the extremal arcs of each pair differ in type by unity.

The first statement of the lemma is an immediate consequence of the definition of the pairs of extremal arcs given in the first paragraph of this section. The last statement in the lemma is a consequence of Lemma 2.1 and the statement in the paragraph immediately preceding this lemma.

Let $p_{i}$ represent the number of pairs of extremal arcs joining the points 1 and 2 , such that one arc of each pair is of type $i$ and the other of type $i-1$. Obviously $p_{i} \geqq 0,(i=1,2,3)$. Let $M_{i}$ represent the number of extremal arcs of type $i$ joining the points 1 and 2 . Then equations $(4: 1)$ and $(4: 2)$, on page 24 of the dissertation [3] referred to above, with $S_{i}=M_{i}$ are easily obtained. Moreover, the in- 
equalities $(4: 3)$ on the same page, with $S_{i}=M_{i},(i=0,1,2,3)$, follow as before except that the $>$ must be replaced by $\geqq$ since now $p_{i} \geqq 0$.

Summarizing the results of the preceding pages we have the following theorem.

THEOREM 3.1. For a calculus of variations problem and a region $R$ satisfying the hypotheses made in $\$ 1$ above, let 1 and 2 represent two fixed points of $R$ such that 2 is not an envelope arc of the family of extremals through 1 . Further let $M_{i}$ represent the number of extremal arcs joining the points 1 and 2 and each of type $i$ and let 3 be the maximum type of each arc. Then the numbers $M_{i},(i=0,1,2,3)$, so defined must satisfy the relations $M_{0} \geqq 1, M_{1} \geqq M_{0}-1, M_{2} \geqq M_{1}-M_{0}+1, M_{3}=M_{2}-M_{1}$ $+M_{0}-1$.

\section{BIBLIOGRAPHY}

1. M. Morse, The Calculus of Variation in the Large, American Mathematical Society Colloquium Publications, vol. 18, New York, 1934.

2. M. Morse, Functional topology and abstract variational theory, Annals of Mathematics, (2), vol. 38 (1937), pp. 386-449.

3. R. H. Bardell, Contributions to the Calculus of Variations, 1933-1937, University of Chicago Press, 1937, pp. 279-312.

University of Wisconsin at MiLwaukee 